\title{
A Clinical Study of the Cognitive Effects of Benzodiazepines in Psychogeriatric Patients
}

Authors

Affiliations

\author{
G. Høiseth ${ }^{1,2}$, L. Tanum ${ }^{3}$, M. Tveito ${ }^{4}$, K. M. Kristiansen ${ }^{4}$, K. Kvande ${ }^{5}$, B. Lorentzen ${ }^{4}$, H. Refsum², J. Bramness ${ }^{2,6}$
}

Affiliation addresses are listed at the end of the article

\author{
Key words \\ - elderly \\ - geriatric psychiatry \\ - drug use \\ - benzodiazepines
}

$\begin{array}{ll}\text { received } & 15.03 .2013 \\ \text { revised } & 03.05 .2013 \\ \text { accepted } & 08.05 .2013\end{array}$

Bibliography

DOI http://dx.doi.org/

10.1055/s-0033-1349131

Published online ahead of print:

5 July 2013

Pharmacopsychiatry 2013;

46: 209-213

(c) Georg Thieme Verlag KG Stuttgart $\cdot$ New York

ISSN 0176-3679

\section{Correspondence}

\section{G. Høiseth, MD, PhD}

Centre for Psychopharmacology Diakonhjemmet Hospital

Forskningsveien 7

P.O. Box 85 Vinderen

0319 Oslo

Norway

Tel.: $+47 / 22 / 498418$

Fax: $+47 / 22 / 029993$

gudrun.hoiseth@fhi.no

\section{Abstract}

$\nabla$

Introduction: Previous studies have shown cognitive impairment in long-term benzodiazepine users compared to non-users. However, little is known about such effects in a population of geriatric psychiatry patients. The aim of this study was to identify differences between benzodiazepine users and non-users on standardized tests of the cognitive fields of learning and memory, executive functions and vigilance, at admittance to a department of geriatric psychiatry. Materials and Methods: Hopkins verbal learning test, Stroop test and digit vigilance test were performed in all patients. Test performances were compared between benzodiazepine users $(\mathrm{n}=168)$ and non-users $(\mathrm{n}=73)$. A multiple linear regression model was used, adjusting for differ-

\section{Introduction}

$\nabla$

A large number of experimental studies has shown that intake of benzodiazepines may lead to cognitive impairment. Cognitive effects comprise many different domains, the most important being learning and memory, executive function and vigilance. Regarding benzodiazepine use, this is shown to affect learning and memory, in particular, and after administration of such drugs, subjects might experience memory reduction and difficulties in learning new material $[1,2]$. It has been questioned whether these negative effects disappear on stable dosing over a longer period of time, owing to development of tolerance. In a meta-analysis of 13 studies, including a total of 384 patients, long-term benzodiazepine users were compared to nonusers [3]. The included subjects were middle aged, averaging 47.6 years and there were significant impairments in all the 12 cognitive domains tested. ent baseline characteristics (years of education, dementia and depression).

Results: No significant differences in test results were found between benzodiazepine users and non-users on 11 out of 12 cognitive tests results. On one of the 12 test results (time used on the digit vigilance test), benzodiazepine users showed better performance compared to non-users $(\beta=-0.20, p=0.032)$. This finding was not statistically significant after Bonferroni correction for multiple testing.

Conclusion: This study of geriatric psychiatry benzodiazepine users did not reveal cognitive impairment compared to non-users on the cognitive areas tested. Other possible negative consequences of benzodiazepine use should, however, also be considered when prescribing drugs to older patients.

Studies on the cognitive effects of benzodiazepine use in the elderly have shown more contradictory results. Some studies indicate that differences between users and non-users are small or absent [4-9]. Some of these studies only used less sensitive tests like the mini mental state examination (MMSE) [7,8], but studies using more complex and comprehensive testing including memory tests also failed to identify cognitive impairment among older benzodiazepine users $[10,11]$. Other studies, however, have documented decreased cognitive function in this population [12-14]. The studies of older benzodiazepine users are mostly population based and therefore include subjects expectedly suffering from fewer maladies than those admitted to hospital wards.

The results from these studies are not necessarily transferable to populations with more marginal cognitive function. To the best of our knowledge, geriatric psychiatry inpatients have not previously been studied with respect to the cognitive 


\begin{tabular}{ll} 
Variable & Bzd users $(\mathbf{n}=\mathbf{1 6 8})$ \\
\hline age $[$ mean \pm SD $(95 \% \mathrm{Cl})]$ & $78.1 \pm 9.1(76.7-79.4)$ \\
\hline no. of females $(\mathrm{n}, \%)$ & $126(75)$ \\
\hline $\mathrm{BMI}\left(\mathrm{kg} / \mathrm{m}^{2}\right)[$ mean $\pm \mathrm{SD}(95 \% \mathrm{Cl})]$ & $24.3 \pm 4.6(23.6-25.0)$ \\
\hline years of education [mean $\pm \mathrm{SD}(95 \% \mathrm{Cl})]$ & $12.8 \pm 3.8(12.3-13.4)$ \\
\hline no. of unmarried patients $(\mathrm{n}, \%)$ & $49(29)$ \\
\hline no.of patients living alone $(\mathrm{n}, \%)$ & $109(65)$ \\
\hline previous psychiatric, admissions [mean $\pm \mathrm{SD}(95 \% \mathrm{Cl})]$ & $1.2 \pm 1.6(0.9-1.4)$ \\
\hline no.of patients with dementia $(\mathrm{n}, \%)$ & $64(38)$ \\
\hline no. of patients with depression $(\mathrm{n}, \%)$ & $96(57)$ \\
\hline no. of patients with psychotic disorders $(\mathrm{n}, \%)$ & $27(16)$ \\
\hline no. of patients with bipolar disorder $(\mathrm{n}, \%)$ & $12(7)$ \\
*Significant value &
\end{tabular}

$\begin{array}{ll}\text { Non-users }(\mathbf{n}=\mathbf{7 3}) & \mathbf{p} \\ 79.8 \pm 8.9(77.7-81.9) & 0.169 \\ 48(66) & 0.160 \\ 24.2 \pm 5.1(23.0-25.4) & 0.904 \\ 11.3 \pm 3.8(10.4-12.2) & 0.006^{*} \\ 13(18) & 0.077 \\ 52(71) & 0.374 \\ 1.0 \pm 1.5(0.6-1.3) & 0.351 \\ 42(58) & 0.026^{*} \\ 20(27) & <0.001^{*} \\ 14(19) & 0.700 \\ 11(15) & 0.061\end{array}$

Table 1 Patient characteristics in the study group (benzodiazepine users) compared to the control group (non-users) and p-value for a difference between the groups. effects of benzodiazepine use. We have shown earlier that a large proportion of patients admitted to a Department of Geriatric Psychiatry were benzodiazepine users [15]. The aim of the current observational study was to investigate whether there are any adverse cognitive effects of benzodiazepines in this population by comparing benzodiazepine users with non-users on standardized cognitive tests concerning the fields of learning and memory, executive functions and vigilance.

\section{Materials and Methods}

This naturalistic observational study included patients admitted to the Department of Geriatric Psychiatry at Diakonhjemmet Hospital, Oslo, Norway. The department receives all patients 60 years or older from the catchment area requiring hospital admission due to psychiatric illnesses. Patients are admitted to one of 3 wards treating affective disorders, severe cognitive impairment and psychosis, respectively. The department has only planned admissions and does not serve as an emergency psychiatric ward. All patients admitted during the period 06.12.2006 to 24.10.2008 (if no exclusion criteria were present) were asked to join the present study, which aimed to investigate all psychopharmacological drug use and potential cognitive consequences. The present article studies the relation between benzodiazepines and cognitive function. The study was approved by the Regional Committee for Research Ethics in Norway and the Norwegian Medicines Agency. All patients signed informed consent before attending the study. Exclusion criteria were: planned electroconvulsive treatment, short expected stay in the department or inability of the patient or a relative to sign informed consent.

Information on any prescribed benzodiazepines was recorded at admission and included diazepam, oxazepam, clonazepam, nitrazepam, alprazolam and flunitrazepam as well as the benzodiazepine-like drugs zopiclone and zolpidem (hereafter referred to as benzodiazepines). Also, serum samples drawn the morning after admittance were analyzed for the benzodiazepine drugs mentioned above. Benzodiazepine users were defined as patients with information of use and/or a positive serum sample. At discharge, the psychiatric diagnoses were recorded.

Use of other psychopharmacological drugs (antidepressants, antipsychotics and mood stabilizers) was also registered.

The morning after admittance, a battery of cognitive tests was carried out by specially trained nurses. The cognitive domains tested were verbal learning and memory (Hopkins verbal learning test (total recall, delayed recall and discrimination ability)) [16], executive functions (Stroop color and word test, number of errors and time used) [17] and vigilance (digit vigilance test, number of errors and time used) [18]. A total of 12 cognitive results ( 4 measuring the time used and 8 measuring number of errors) was obtained in each patient if all tests were completed. In addition, an MMSE screening was performed.

As an indication of psychopathological symptoms at the time of testing, Montgomery-Asberg depression rating scale (MADRS), confusion assessment method (CAM, measuring delirium) and a physician's clinical global impression (CGI) of the patient's condition, were performed.

Serum concentrations of benzodiazepines were analysed using an ultra-performance liquid chromatography-mass spectrometry (UPLC-MS/MS) method developed for routine therapeutic drug monitoring at the Centre for Psychopharmacology, Diakonhjemmet Hospital [15].

IBM SPSS ${ }^{\circledR}$ Software version 19.0 (SPSS Inc., Chicago, IL, USA) was used for the statistical analyses. For descriptive analyses of continuous variables, means \pm standard deviation (SD), were reported. For continuous variables compared between 2 groups, means \pm SD [95\% confidence interval $(\mathrm{CI})$ for the mean], were reported. The frequency distribution was reported for categorical variables. For patient's characteristics, Student's t-test was used to compare continuous variables, and Fischer's exact test was used to compare categorical variables.

Test performances were compared between benzodiazepine users and non-users first using Student's t-test. To adjust for variables that differed between benzodiazepine users and nonusers, the test performances were also compared using a multiple linear regression analysis with years of education, presence of dementia and presence of depression as covariates. We chose to use these covariates as these were the only variables which differed significantly between benzodiazepine users and nonusers. In this analysis, the effects of education, dementia and depression were also assessed. Standardized $\beta$ coefficients with p-values are reported.

\section{Results}

$\nabla$

241 patients (174 female, 67 male) were included in the study. Of these, 168 ( 126 female, 42 male) were identified as benzodiazepine users and constitute the study group. The remaining 73 patients ( 48 female, 25 male) were not benzodiazepine users and constitute the control group. $\odot$ Table 1 gives the patient characteristics. A diagnosis of dementia was seen in 106 (44\%) of the patients, unipolar depression was seen in 116 (48\%) while bipolar disorder and psychotic disorders was seen in 23 (10\%) and 41 (17\%), respectively. Benzodiazepine users had significantly less dementia $(p=0.026)$ and more depression $(p<0.001)$, compared 
to non-users. The other diagnoses did not differ significantly between the groups. Benzodiazepine users also had more years of education $(\mathrm{p}=0.006)$, compared to non-users. Other patient characteristics were not significantly different between the 2 groups ( Table 1). The mean MMSE value in the total patient material was $24.2 \pm 0.40$ and 30 patients showed an MMSE score below 18 .

The number of users and doses of each benzodiazepine is shown in Table 2. Few serum samples showed concentrations above those corresponding to use of recommended doses, except for users of zopiclone.

All the cognitive tests could not be completed by all patients, and for the Hopkins verbal learning tests, complete results were obtained in 123 benzodiazepine users and 45 non-users. The Stroop colour/word test was completely performed in 114 users and 46 non-users and the digit vigilance test was performed completely in 91 users and 35 non-users. All tests are seen in $\odot$ Table 3 and $\odot$ Fig. 1.

The most frequently used other psychopharmacological drugs are shown in $\bullet$ Table 4 . This table shows those drugs used by more than 9 patients in any of the groups. Among these, only the number of chlorprothixen users differed significantly between the groups ( $\bullet$ Table 4 ).

Using Student's t-test, benzodiazepine users performed significantly better compared to non-users on 3 out of 12 tests. On the Hopkins verbal learning test, total recall, a mean result of $18.9 \pm 6.7$ (17.7-20.1) words was seen in benzodiazepine users, while non-users showed a mean result of $15.2 \pm 6.6(13.2-17.2)$ $(p=0.002)$ words. The same situation was seen for the Hopkins verbal learning test, delayed recall $[5.7 \pm 3.6(5.0-6.3)$ vs. $4.2 \pm 3.2$ (3.2-5.2) words, $\mathrm{p}=0.018$ ] and the digit vigilance test (time

Table 2 Number of users, doses and number of patients with high serum concentrations, for each benzodiazepine in the study group $(n=168)$. Note that the same patient might use more than one drug.

\begin{tabular}{|c|c|c|c|}
\hline & $\begin{array}{l}\text { Bzd users, } \\
\text { n (\%) }\end{array}$ & $\begin{array}{l}\text { Dose }(\mathrm{mg}) \text {, } \\
\text { mean } \pm S D\end{array}$ & $\begin{array}{l}\text { High serum } \\
\text { concentration }{ }^{1} \text {, } \\
\text { n (\%) }\end{array}$ \\
\hline diazepam & $63(38)$ & $7.3 \pm 4.03$ & $4(6.3)$ \\
\hline oxazepam & $90(54)$ & $21.2 \pm 15.6$ & 0 \\
\hline zopiclone & $102(61)$ & $6.9 \pm 2.6$ & $26(25)$ \\
\hline zolpidem & $14(8.3)$ & $7.3 \pm 2.9$ & 0 \\
\hline alprazolam & $4(2.4)$ & $2(2-2)^{2}$ & $1(25)$ \\
\hline nitrazepam & $18(11)$ & $3.8 \pm 1.5$ & 0 \\
\hline clonazepam & $13(7.7)$ & $0.9 \pm 0.2$ & 0 \\
\hline flunitrazepam & $3(1.8)$ & $1(1-1)^{2}$ & 0 \\
\hline
\end{tabular}

used) $[750 \pm 226$ (702-797) vs. 887 \pm 352 (764-1009) seconds, $\mathrm{p}=0.041]$ ( Table 3 ). Also on the MMSE screening, benzodiazepine users showed improved performance [25 \pm 5.4 (24.1$25.8)$ ] compared to non-users [22.4 $\pm 6.6(20.8-24.1), \mathrm{p}=0.008$ ] On the rest of the tests, there was no significant difference between the performance of benzodiazepine users and nonusers, using Student's t-test.

To adjust for differences between the 2 groups, the tests were also performed using a multiple linear regression model. Only those variables which significantly differed between benzodiazepine users and non-users (education, dementia and depression) ( $\bullet$ Table 1 ) were inserted in the model as covariates. In these analyses, the group of benzodiazepine users performed significantly better compared to non-users only on the digit vigilance test (time used) $(\beta=-0.20, p=0.032)$. However, when the number of errors on the digit vigilance test was assessed, there was no difference between the groups. If considering the problem of multiple testing, only p-values below 0.004 would be considered significant, according to the Bonferroni method [19]. In this case, there would be no significant difference in performance on any of the tests between benzodiazepine users and nonusers. The results are shown in $\odot$ Table 3 and $\bullet$ Fig. 1. $\bullet$ Fig. 1 shows the 3 different Hopkins verbal learning tests, while the remaining tests are shown in $\bullet$ Table 3.

The 3 patient characteristics which differed significantly between benzodiazepine users and non-users (education, dementia and depression) were all related to cognitive function. This was seen in the multiple linear regression model, and results are shown only for the Hopkins verbal learning test (total recall). High education resulted in improved performance $(\beta=0.24, p<0.001)$, compared to low education. Patients with a diagnosis of dementia showed reduced performance $(\beta=-0.37$, $\mathrm{p}<0.001$ ), compared to patients without this diagnosis. Finally, patients with a diagnosis of depression showed improved performance $(\beta=0.30, p<0.001)$, compared to patients without this diagnosis.

The CAM and CGI values did not differ significantly between the 2 groups $(p=0.636$ and $p=0.068$, respectively). The MADRS score, however, was significantly higher in the benzodiazepine users $(\mathrm{p}=0.001)$, corresponding to more presence of depression in this group. This test was not completely performed in 36 benzodiazepine users and 29 non-users.

\section{Discussion}

This study among patients admitted to a department of geriatric psychiatry showed that benzodiazepine users were not more

\section{Test}

Stroop colour (time in s)

Stroop words (time in s)

Stroop colour/words (time in s)

Stroop colour (number of errors)

Stroop words (number of errors)

Stroop color/words (number of errors)

Stroop total number of errors

digit vigilance test (total time in s)

digit vigilance test, total errors

*Significant value (using Student's t-test)

\begin{tabular}{lll}
$\begin{array}{l}\text { Bzd users }(\mathbf{n}=\mathbf{1 6 8}), \\
\text { mean } \pm \text { SD }(\mathbf{9 5} \% \mathrm{Cl})\end{array}$ & $\begin{array}{l}\text { Non-users }(\mathbf{n}=\mathbf{7 3}), \\
\text { mean } \pm \text { SD }(\mathbf{9 5} \% \mathbf{C l})\end{array}$ & p-value \\
$65.5 \pm 67.0(53.1-78.0)$ & $82.1 \pm 66.9(62.3-102)$ & 0.160 \\
$31.0 \pm 17.2(27.8-34.2)$ & $31.6 \pm 10.5(28.5-34.7)$ & 0.778 \\
$133.8 \pm 68.7(118.2-192.0)$ & $155.1 \pm 93.1(118.2-192.0)$ & 0.277 \\
$1.4 \pm 2.7(0.9-1.9)$ & $2.3 \pm 4.4(0.9-3.6)$ & 0.218 \\
$0.1 \pm 0.5(0-0.20)$ & $0.2 \pm 1.0(0-0.48)$ & 0.633 \\
\hline $5.1 \pm 5.4(4.0-6.3)$ & $6.7 \pm 8.3(3.4-10)$ & 0.365 \\
$5.9 \pm 6.2(4.6-7.2)$ & $7.1 \pm 8.6(3.8-10.5)$ & 0.476 \\
$749.7 \pm 226.5(702.2-797.1)$ & $886.5 \pm 351.9(763.7-1009.3)$ & $0.041^{*}$ \\
$12.6 \pm 17.1(9.0-16.2)$ & $13.9 \pm 17.3(7.9-20)$ & 0.702
\end{tabular}

Non-users $(n=73)$, mean \pm SD $(95 \% \mathrm{Cl})$

$2.3 \pm 4.4(0.9-3.6)$

$0.2 \pm 1.0(0-0.48)$

$6.7 \pm 8.3(3.4-10)$

$886.5 \pm 351.9(763.7-1009.3)$

$13.9 \pm 17.3(7.9-20)$
Table 3 Mean \pm SD $(95 \%$ Cl for the mean) values for results of cognitive tests (excluding Hopkins verbal learning tests, which are shown in 1 Fig. 1), compared between benzodiazepine users (study group) and non-users (control group). The p-value for a difference between the groups is also given. 


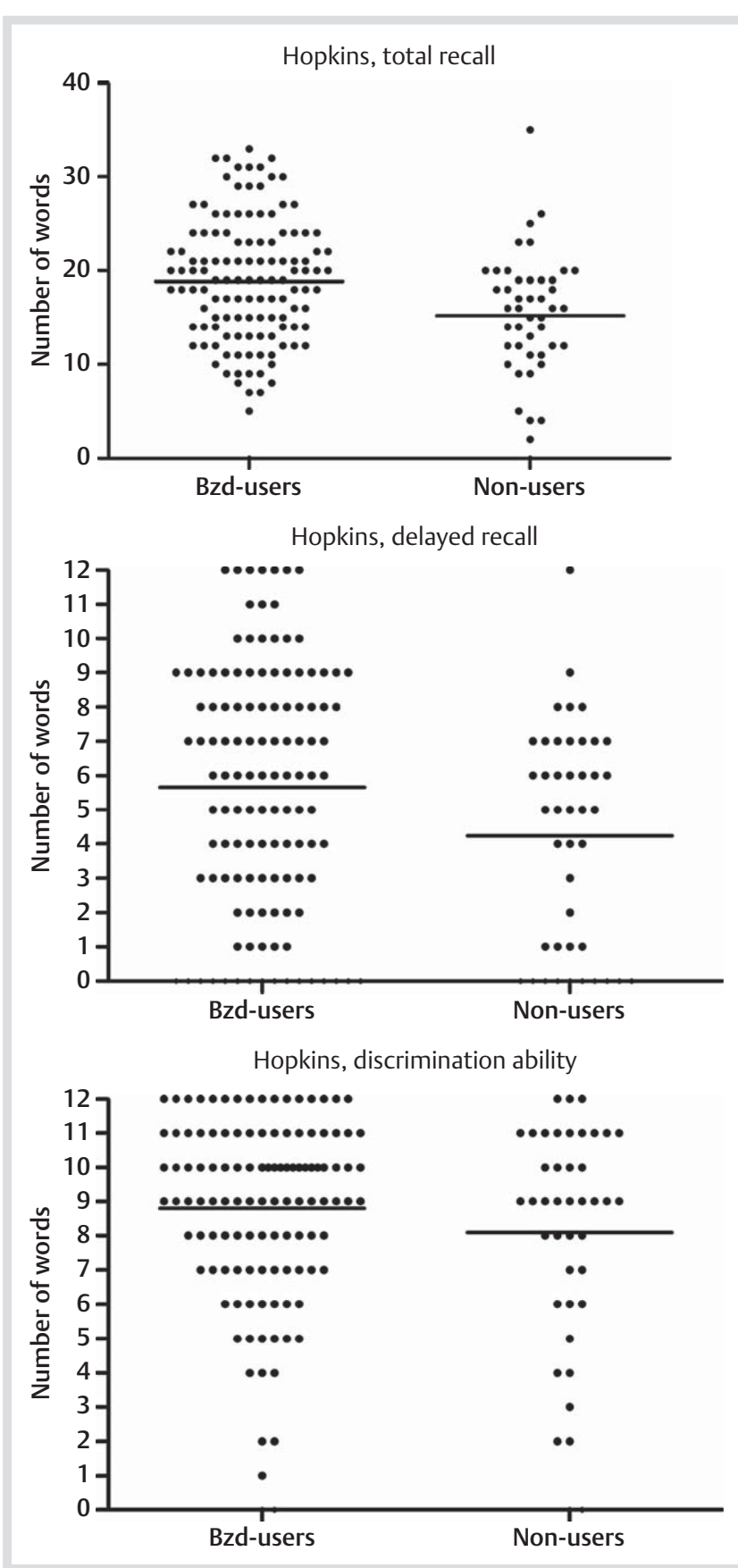

Fig. 1 Individual results for benzodiazepine (bzd) users and non-users, respectively, for the Hopkins verbal learning tests (total recall, delayed recall and discrimination ability).

impaired than non-users on the cognitive fields of learning and memory, executive functions and vigilance. On one out of 12 test results, benzodiazepine users actually performed better compared to non-users. This difference, however, was not significant when multiple testing was taken into consideration.

These results negate the general understanding of reduced cognitive function following benzodiazepine use, which is based partly on the meta-analysis by Barker and co-workers [3], and partly on some of the earlier studies of older people [12-14]. These different results could be explained by several differences in the study populations. Firstly, earlier studies have investigated much healthier elders. For example, a study finding reduced cognitive function in older benzodiazepine users
Table 4 Number of users of the most frequently used other psychopharmacological drugs in benzodiazepine users vs. non-users. Drugs used by $>9$ patients in any group are shown. P-values for differences between groups.

\begin{tabular}{|llcl|} 
& $\begin{array}{l}\text { Bzd users } \\
(\mathbf{n = 1 6 8 )} \mathbf{n}(\%)\end{array}$ & $\begin{array}{l}\text { Non-users } \\
(\mathbf{n = 7 3 )} \mathbf{n}(\%)\end{array}$ & $\mathbf{p}$ \\
\hline mirtazapine & $32(19)$ & $7(10)$ & 0.086 \\
\hline olanzapine & $23(14)$ & $11(15)$ & 0.841 \\
\hline chlorprothixen & $22(13)$ & $2(3)$ & $0.017^{*}$ \\
\hline escitalopram & $16(10)$ & $5(7)$ & 1.000 \\
\hline citalopram & $13(8)$ & $2(3)$ & 0.562 \\
\hline lithium & $12(7)$ & $3(4)$ & 0.563 \\
\hline haloperidol & $11(7)$ & $8(11)$ & 0.298 \\
\hline *Significant value & & & \\
\hline
\end{tabular}

reported a mean MMSE level of 28 [14], which is higher than that seen in our group (mean MMSE score 24.2). Only one study of long-term benzodiazepine effects included elderly patients admitted to hospital. These had cognitive functions comparable to those of the patients included in our study [8] and the investigation found no relation between benzodiazepine use and cognitive function measured by MMSE. Thus, the evidence so far suggests less cognitive effect of long-term benzodiazepine use in patients who are already cognitively impaired, possibly because the effect of the benzodiazepines is smaller than the cognitive effects caused by the different maladies.

As our study group had a higher frequency of benzodiazepine use than the average Norwegian elderly population [20] and reported in earlier studies [7], we would expect there to be a high sensitivity in picking up on the cognitive effects of benzodiazepine use. However, our study included patients using low doses of benzodiazepines (mean diazepam dose $7.3 \mathrm{mg}$ ). As a dose-response curve is well documented for benzodiazepines [21], reduced effects would be expected from such doses, compared to, for example, the meta-analysis by Barker et al. (mean diazepam dose $17.3 \mathrm{mg}$ ) [3].

The strength of our study was the relatively large number of subjects and the performance of sensitive, well-validated cognitive tests. Also, unlike most other studies, we measured serum concentrations to validate the information of benzodiazepine use. One weakness of the study was the lack of information on the duration of benzodiazepine use. We could not, therefore, be sure if patients were long-term users. However, this would not be expected to bias the results, as short-term use would tend to produce more pronounced impairment. We do not know if all the patients actually used their benzodiazepines every day, but the fact that almost all the prescribed drugs were detected in serum suggests that frequent use was widespread.

The use of Bonferroni correction on the present data could be debated, as the 12 different results descend from only 3 different cognitive tests. If not correcting for multiple testing, there is still no evidence of reduced performance in benzodiazepine users. The fact that the benzodiazepine users had more years of education, less dementia and more often had major depression compared to the non-users was an interesting observation. Previous studies have often excluded patients with cognitive failure $[7,12]$, but in our study, we aimed to describe a naturalistic population of geriatric psychiatry patients, where dementia and major depression are frequent diagnoses. When the total material of both benzodiazepine users and non-users was studied, there was, as expected, a strong effect of the diagnosis dementia and also of educational level on results of the cognitive tests (complete data not shown). This supports the view that factors 
other than benzodiazepine use are more important for cognitive function in geriatric psychiatry inpatients, although our study was not designed to investigate this in detail. An especially intriguing result was the positive relationship between major depression and cognitive function. This could be explained by the control group in this analysis, which contained patients with diagnoses other than depression, such as, dementia which may have had more of an effect on cognitive function.

Although this study suggests that the cognitive effects of lowdose benzodiazepine use in geriatric psychiatry patients might be less pronounced than previously anticipated, other adverse effects of benzodiazepine use in the elderly can be of more importance. Use of benzodiazepines might lead to dizziness and daytime sleepiness $[8,22]$. The increased risk of falling accidents and hip fractures in elderly benzodiazepine users is well documented [23-27]. These factors need to be kept in mind when prescribing medicines to this patient population.

\section{Acknowledgements}

$\nabla$

None

\section{Conflict of Interest}

The authors declare no conflicts of interest.

\section{Affiliations}

${ }^{1}$ Norwegian Institute of Public Health, Division of Forensic Medicine and Drug Abuse Research, Oslo, Norway

${ }^{2}$ Center for Psychopharmacology, Diakonhjemmet Hospital, Oslo, Norway

${ }^{3}$ Department of Research \& Development in Psychiatry, Akershus University Hospital and University of Oslo, Norway

${ }^{4}$ Department of Geriatric Psychiatry, Diakonhjemmet Hospital, Oslo, Norway

${ }^{5}$ Norwegian Medicines Agency, Oslo, Norway

${ }^{6}$ Norwegian Centre for Addiction Research, University of Oslo, Norway

\section{References}

1 Roache JD, Griffiths RR. Comparison of triazolam and pentobarbital: performance impairment, subjective effects and abuse liability. J Pharmacol Exp Ther 1985; 234: 120-133

2 Evans SM, Funderburk FR, Griffiths RR. Zolpidem and triazolam in humans: behavioral and subjective effects and abuse liability. J Pharmacol Exp Ther 1990; 255: 1246-1255

3 Barker MJ, Greenwood KM, Jackson M et al. Cognitive effects of longterm benzodiazepine use: a meta-analysis. CNS Drugs 2004; 18: 37-48

4 Allard J, Artero S, Ritchie K. Consumption of psychotropic medication in the elderly: a re-evaluation of its effect on cognitive performance. Int J Geriatr Psychiatry 2003; 18: 874-878

5 Lagnaoui R, Tournier M, Moride $Y$ et al. The risk of cognitive impairment in older community-dwelling women after benzodiazepine use. Age Ageing 2009; 38: 226-228

6 Pat MM, Weiss RT, Sandor P et al. Cognitive effects of long-term benzodiazepine use in older adults. Hum Psychopharmacol 2003; 18: 51-57
7 Puustinen J, Nurminen J, Lopponen $M$ et al. Use of CNS medications and cognitive decline in the aged: a longitudinal population-based study. BMC Geriatr 2011; 11: 70

8 Puustinen J, Nurminen J, Kukola $M$ et al. Associations between use of benzodiazepines or related drugs and health, physical abilities and cognitive function: a non-randomised clinical study in the elderly. Drugs Aging 2007; 24: 1045-1059

9 Verdoux $H$, Lagnaoui $R$, Begaud $B$. Is benzodiazepine use a risk factor for cognitive decline and dementia? A literature review of epidemiological studies. Psychol Med 2005; 35: 307-315

10 van VP, van der Mast RC, van den Broek $M$ et al. Use of benzodiazepines, depressive symptoms and cognitive function in old age. Int J Geriatr Psychiatry 2009; 24: 500-508

11 Bierman EJ, Comijs HC, Gundy CM et al. The effect of chronic benzodiazepine use on cognitive functioning in older persons: good, bad or indifferent? Int J Geriatr Psychiatry 2007; 22: 1194-1200

12 Foy $A, O$ 'Connell $D$, Henry $D$ et al. Benzodiazepine use as a cause of cognitive impairment in elderly hospital inpatients. J Gerontol A Biol Sci Med Sci 1995; 50: M99-M106

13 Hanlon JT, Horner RD, Schmader KE et al. Benzodiazepine use and cognitive function among community-dwelling elderly. Clin Pharmacol Ther 1998; 64: 684-692

14 Paterniti S, Dufouil C, Alperovitch A. Long-term benzodiazepine use and cognitive decline in the elderly: the Epidemiology of Vascular Aging Study. J Clin Psychopharmacol 2002; 22: 285-293

15 Hoiseth G, Kristiansen KM, Kvande K et al. Benzodiazepines in geriatric psychiatry - what doctors report and patients actually use. Drugs Aging 2013; 30: 113-118

16 Brandt J, Benedict RHB. Hopkins verbal learning test - revised. Odessa, FL: Psychological Assessment Resources; 2001

17 Stroop J. Studies of interference in serial verbal reaction. J Exp Psychol 1935; 18: 643-662

18 Lewis LJ. Digit Vigilance Test: Professional users guide. Lutz, FL: Psychological Assessment Resources; 1995

19 Streiner DL, Norman GR. Correction for multiple testing: is there a resolution? Chest 2011; 140: 16-18

20 Neutel CI, Skurtveit S, Berg C. What is the point of guidelines? Benzodiazepine and z-hypnotic use by an elderly population. Sleep Med 2012; 13: 893-897

21 Bramness JG, Skurtveit S, Morland J. Clinical impairment of benzodiazepines - relation between benzodiazepine concentrations and impairment in apprehended drivers. Drug Alcohol Depend 2002; 68: 131-141

22 Glass J, Lanctot KL, Herrmann $N$ et al. Sedative hypnotics in older people with insomnia: meta-analysis of risks and benefits. BMJ 2005; 331: 1169

23 Landi F, Cesari M, Russo A et al. Benzodiazepines and the risk of urinary incontinence in frail older persons living in the community. Clin Pharmacol Ther 2002; 72: 729-734

24 Finkle WD, Der JS, Greenland $S$ et al. Risk of fractures requiring hospitalization after an initial prescription for zolpidem, alprazolam, lorazepam, or diazepam in older adults. J Am Geriatr Soc 2011; 59: 1883-1890

25 Sylvestre MP, Abrahamowicz M, Capek R et al. Assessing the cumulative effects of exposure to selected benzodiazepines on the risk of fallrelated injuries in the elderly. Int Psychogeriatr 2011; 1-10

26 Titler MG, Shever LL, Kanak MF et al. Factors associated with falls during hospitalization in an older adult population. Res Theory Nurs Pract 2011; 25: 127-148

27 Nurminen J, Puustinen J, Piirtola $M$ et al. Psychotropic drugs and the risk of fractures in old age: a prospective population-based study. BMC Public Health 2010; 10: 396 